\title{
Hedging portfolio loss derivatives with CDSs*
}

\author{
Areski Cousin ${ }^{\dagger}$ and Monique Jeanblanc ${ }^{\ddagger}$
}

February 22, 2010

\begin{abstract}
In this paper, we consider the hedging of portfolio loss derivatives using single-name credit default swaps as hedging instruments. The hedging issue is investigated in a general pure jump dynamic setting where default times are assumed to admit a joint density. In a first step, we compute default intensities adapted to the global filtration of defaults. In particular, we stress the impact of a default event on the price dynamics of non-defaulted names. In a two defaults setting, we also fully describe the hedging of a loss derivative with single name instruments. The methodology can be applied recursively to the case of a multidefault setting. We completely characterize the hedging strategies for general $n$-dimensional credit portfolios when default times are assumed to be ordered. The computation of the hedging strategies does not require any Markovian assumption.
\end{abstract}

\section{Introduction}

The hedging of loss derivatives such as CDO tranches or basket default swaps is a prominent risk-management issue especially given the recent revision of the Basel II regulation on calculation of trading book capital requirement. Indeed, according to Basel Committee Guidelines for computing capital for incremental risk in the trading book, July 2009, "for trading book risk positions that are typically hedged via dynamic hedging strategies, a rebalancing of the hedge within the liquidity horizon of the hedged position may be recognized as a risk mitigation. Moreover, any residual risks resulting from dynamic hedging strategies must be reflected in the capital charge." As a result, the performance and efficiency of underlying hedging methods is going to have a direct impact on the amount of capital required for loss derivatives. Cousin and Laurent (2010) discuss various issues related to the use of models in designing hedging strategies for CDO tranches and back-testing or assessing hedging performance.

In this paper, we consider the hedging of loss derivatives using single-name credit default swaps as hedging instruments. The hedging issue is investigated in a general pure jump setting where default times are assumed to admit a joint density which is the only input of the model so that our results can be considered as model independent - and we compute default intensities adapted to the global filtration of defaults. We check that, if CDSs on each default are traded, the market is complete. The hedging strategies can be found by identifying the terms associated with the fundamental default martingales.

We extend some recent results by Laurent, Cousin and Fermanian (2007) and Cousin, Jeanblanc and Laurent (2009). In particular, we stress the impact of a default event on the price dynamics of non-defaulted names. Moreover, in a two defaults setting, we fully describe the hedging of a loss derivative with single name instruments. The generalization to a multidefault setting can be done following the same methodology. Furthermore, we are able to completely characterize the hedging strategies in single-name CDS for general $n$-dimensional credit portfolios when default times are assumed to be ordered. The computation of the hedging strategies

\footnotetext{
* This research is a part of CRIS programm

†Département de Mathématiques, Equipe analyse et probabilité, Université d'Evry

‡Département de Mathématiques, Equipe analyse et probabilité, Université d’Evry; Institut Europlace de Finance
} 
does not require any Markovian assumption.

The paper is organized as follows. The first section aims at presenting the general setting of the model and it recalls the predictable representation theorem. In the second section, we investigate the case where only one name is considered. In particular, we exhibit the intensity of the default time and the dynamics of CDS prices. Section three is devoted to the case where the credit portfolio is composed of two names. The extension to a multivariate setting can be done using a recursive procedure. In particular, we highlight the contagion effect occurring at default time of one of the two names on the CDS price dynamics of the other name. We also compute the dynamics of the hedging strategies at any time in all the possible default situations. In section four, we consider the hedging of a loss derivatives written on a general $n$-dimensional portfolio. We stress that when default times are assumed to be ordered, i.e., CDS are $k$ th-todefault swaps, the hedging strategies can be simply characterized as the solutions of a linear system.

\section{Mathematical tools: the general case}

In what follows, we consider $n$ default times $\tau_{i}, i=1, \ldots, n$, that is, non-negative and finite random variables constructed on the same probability space $(\Omega, \mathcal{G}, \mathbb{P})$. For any $i=1, \ldots, n$, we denote by $\left(H_{t}^{i}=\mathbb{1}_{\tau_{i} \leq t}, t \geq 0\right)$ the $i$-th default process, and by $\mathcal{H}_{t}^{i}=\sigma\left(H_{s}^{i}, s \leq t\right)$ the natural filtration of $H^{i}$ (after completion and regularization on right). We introduce $\mathbb{H}$, the filtration generated by the processes $H^{i}, i=1, \ldots, n$, defined as $\mathbb{H}=\mathbb{H}^{1} \vee \ldots \vee \mathbb{H}^{n}$, i.e., $\mathcal{H}_{t}=\vee_{i=1}^{n} \mathcal{H}_{t}^{i}$.

We assume that $G\left(t_{1}, \ldots, t_{n}\right):=\mathbb{P}\left(\tau_{1}>t_{1}, \ldots, \tau_{n}>t_{n}\right)$ is twice differentiable with respect to $\left(t_{1}, \ldots, t_{n}\right)$ and that $G$ and its derivatives do not vanish. Then, as we shall prove in the next section, for any $i=1, \ldots, n$, there exists a non-negative $\mathbb{H}$-adapted process $\left(\lambda_{t}^{i}, t \geq 0\right)$ such that the process

$$
M_{t}^{i}:=H_{t}^{i}-\int_{0}^{t} \lambda_{s}^{i} d s
$$

is an $\mathbb{H}$-martingale. The process $\lambda^{i}$ is called the $\mathbb{H}$-intensity of $\tau_{i}$. This process vanishes after $\tau_{i}$ (otherwise, after $\tau_{i}$, the martingale $M^{i}$ would be continuous and decreasing) and can be written $\lambda_{t}^{i}=\left(1-H_{t}^{i}\right) \widetilde{\lambda}_{t}^{i}$ for some $\mathbb{H}^{1} \vee \ldots \vee \mathbb{H}^{i-1} \vee \mathbb{H}^{i+1} \vee \ldots \vee \mathbb{H}^{n}$-adapted process $\tilde{\lambda}^{i}$. In terms of the process $\tilde{\lambda}^{i}$, one has

$$
M_{t}^{i}=H_{t}^{i}-\int_{0}^{t \wedge \tau_{i}} \widetilde{\lambda}_{s}^{i} d s=H_{t}^{i}-\int_{0}^{t}\left(1-H_{s}^{i}\right) \widetilde{\lambda}_{s}^{i} d s .
$$

In particular, denoting by $\tau_{(i)}$ the ranked sequence of default times, the process $\widetilde{\lambda}^{1}$ is deterministic on the time interval $\left[O, \tau_{(1)}\left[\right.\right.$, is a deterministic function of $\tau_{(1)}$ on the time interval $\left[\tau_{(1)}, \tau_{(2)}[\right.$, and a deterministic function of $\tau_{(j)}, j \leq i$ on the time interval $\left[\tau_{(i)}, \tau_{(i+1)}[\right.$. In particular, the value of the intensity depends not only of the number of default occurred in the past, but also on the time where the default have take place, which is more realistic.

The following predictable representation theorem holds true (see Brémaud [5]).

Theorem 1.1 Let $B \in \mathcal{H}_{T}$ be an integrable random variable. Then, there exists $\mathbb{H}$-predictable processes $\vartheta^{i}, i=1, \ldots, n$ such that

$$
B=\mathbb{E}(B)+\sum_{i=1}^{n} \int_{0}^{T} \vartheta_{s}^{i} d M_{s}^{i}
$$

and $\int_{0}^{T}\left|\vartheta_{s}^{i}\right| \lambda_{s}^{i} d s<\infty$, and these processes are unique.

Due to the integrability assumption and the predictable property of the $\vartheta$ 's, the processes $\int_{0}^{t} \vartheta_{s}^{i} d M_{s}^{i}$ are $\mathbb{H}$-martingales.

We shall in a first part present computations for the intensity in terms of the density of $\tau$ in the case $n=1$. Then, we shall study the case $n=2$ and we determine the hedging strategy of any payoff, when the hedging instruments are CDSs. The methodology can be easily extended to other hedging instruments, as defaultable zero-coupons, digital CDSs. The multidefault case can be studied along the same lines. For simplicity, we restrict our attention to the particular situation of ranked times. 


\section{The single default case}

We present some well known results concerning the dynamics of a CDS written on a single default, working in the filtration $\mathbb{H}^{1}$ (as we shall see in the next section, the dynamics of the CDS with the same recovery) will be different in the filtration taking into account the knowledge of other defaults).

\subsection{Some important martingales}

We recall some well known results (see Dellacherie [9] and Bielecki and Rutkowski [2]). Here, $\tau$ is a non-negative random variable on the probability space $(\Omega, \mathcal{G}, \mathbb{P})$ with survival function $G(t):=\mathbb{P}(\tau>t)=1-\mathbb{P}(\tau \leq t)=1-F(t)$ where $F$ is the cumulative distribution function of $\tau$. We assume that $G(t)>0, \forall t$, and that $G$ is differentiable, i.e., that $\tau$ admits a density $f$, so that $G^{\prime}(t)=-f(t)$. The filtration is $\mathbb{H}=\mathbb{H}^{1}$.

Proposition 2.1 For any (integrable) random variable $X$

$$
\mathbb{1}_{t<\tau} \mathbb{E}_{\mathbb{P}}\left(X \mid \mathcal{H}_{t}\right)=\mathbb{1}_{t<\tau} \frac{1}{G(t)} \mathbb{E}_{\mathbb{P}}\left(X \mathbb{1}_{t<\tau}\right)
$$

and for any Borelian (bounded) function $h$

$$
\mathbb{E}_{\mathbb{P}}\left(h(\tau) \mid \mathcal{H}_{t}\right)=\mathbb{1}_{\tau \leq t} h(\tau)-\mathbb{1}_{t<\tau} \frac{1}{G(t)} \int_{t}^{\infty} h(u) d G(u) .
$$

The process $\left(M_{t}, t \geq 0\right)$ defined as

$$
M_{t}=H_{t}-\int_{0}^{\tau \wedge t} \frac{f(s)}{G(s)} d s=H_{t}-\int_{0}^{t}\left(1-H_{s}\right) \frac{f(s)}{G(s)} d s
$$

is a $(\mathbb{P}, \mathbb{H})$-martingale. In other terms, the $\mathbb{H}$-intensity of $\tau$ is $\left(1-H_{t}\right) \tilde{\lambda}(t)$ where $\tilde{\lambda}$ is the deterministic function $\widetilde{\lambda}(t)=\frac{f(t)}{G(t)}$.

Note that the survival probability $G$ can be expressed in terms of the deterministic function $\tilde{\lambda}$ : indeed we have proved that

$$
\widetilde{\lambda}(t)=f(t) / G(t)=-G^{\prime}(t) / G(t) .
$$

Solving this ODE with initial condition $G(0)=1$ leads to

$$
G(t)=\mathbb{P}(\tau>t)=\exp \left(-\int_{0}^{t} \tilde{\lambda}(u) d u\right) .
$$

Note that $\tilde{\lambda}(t) d t=\mathbb{P}(\tau \in d t \mid \tau>t)$. The default intensity can be interpreted as the instantaneous conditional default probability given that default has not yet occurred.

\subsection{Price of a traditional single-name CDS}

We assume that the underlying market model is arbitrage-free, meaning that it admits a martingale measure $\mathbb{Q}$ (not necessarily unique) equivalent to the historical probability $\mathbb{P}$. The risk-neutral survival probability $G^{\mathbb{Q}}$ is defined as

$$
G^{\mathbb{Q}}(t)=\mathbb{Q}(\tau>t)=\int_{t}^{+\infty} f^{\mathbb{Q}}(s) d s
$$

and the $(\mathbb{Q}, \mathbb{H})$-intensity is $\lambda_{t}^{\mathbb{Q}}=\mathbb{1}_{t<\tau} f^{\mathbb{Q}}(t) / G^{\mathbb{Q}}(t)$. We shall simply denote by $G$ (resp. $\lambda$ ) the survival function (resp. the intensity), and forget the superscript $\mathbb{Q}$. The expectation $\mathbb{E}$ is computed under the probability $\mathbb{Q}$.

We denote by $B$ the savings account, henceforth the price process of any tradeable security, paying no coupons or dividends, is a $(\mathbb{Q}, \mathbb{H})$-martingale, when discounted by $B$. The price of an asset paying dividends is

$$
B_{t} \mathbb{E}\left(\int_{t}^{T} B_{s}^{-1} d D_{s} \mid \mathcal{F}_{t}\right)
$$


where $D$ represents the cumulative dividend. In that case, the discounted cum-dividend price

$$
V_{t}^{\text {cum }} B_{t}^{-1}=\mathbb{E}\left(\int_{t}^{T} B_{s}^{-1} d D_{s} \mid \mathcal{F}_{t}\right)+\int_{0}^{t} B_{s}^{-1} d D_{s}
$$

is a martingale. As usual, $B$ is given by

$$
B_{t}=\exp \left(\int_{0}^{t} r_{u} d u\right), \quad \forall t \in \mathbb{R}_{+},
$$

where the short-term interest rate $r$ is a stochastic process.

Let us recall that a credit default swaps is a bilateral contract involving a protection seller and a protection buyer. We consider a CDS maturing at time $T$. If a default event occurs at time $\tau<T$, then the protection seller delivers to the protection buyer the unrecovered portion of the loss $\delta(\tau)^{1}$ where $\delta$ is a deterministic function. As for the premium leg, we assume for simplicity that the fee is paid to the protection seller in continuous time, i.e., the amount $\kappa d t$ is paid by the protection buyer during the time interval $d t$, till time $\tau \wedge T$. The time- $t$ market value of a CDS with payment at default $\delta$ and with a contractual spread $\kappa$ is equal to

$$
V_{t}(\kappa)=D_{t}-\kappa P_{t},
$$

where $D_{t}$ and $P_{t}$, the default leg and the premium leg, are given by

$$
\begin{aligned}
D_{t} & =B_{t} \mathbb{E}\left(B_{\tau}^{-1} \delta(\tau) \mathbb{1}_{\{t<\tau \leq T\}} \mid \mathcal{H}_{t}\right) \\
P_{t} & =B_{t} \mathbb{E}\left(\int_{t \wedge \tau}^{T \wedge \tau} B_{u}^{-1} d u\right)
\end{aligned}
$$

and the cum-dividend price is

$$
V_{t}^{\text {cum }}=B_{t} \mathbb{E}\left(B_{\tau}^{-1} \delta(\tau) \mathbb{1}_{\tau \leq T}-\kappa \int_{0}^{T \wedge \tau} B_{u}^{-1} d u \mid \mathcal{H}_{t}\right) .
$$

In the case of a zero interest rate,

$$
\begin{aligned}
V_{t} & =\mathbb{1}_{t<\tau} \mathbb{E}\left(\delta(\tau) \mathbb{1}_{\tau \leq T}-\kappa((T \wedge \tau)-t) \mid \mathcal{H}_{t}\right), \\
V_{t}^{\text {cum }} & =\mathbb{E}\left(\delta(\tau) \mathbb{1}_{\tau \leq T}-\kappa(T \wedge \tau) \mid \mathcal{H}_{t}\right) .
\end{aligned}
$$

It is worthwhile to note that the price dynamics is not a martingale under the risk-neutral probability, despite the fact that the interest rate is null. However, the cum-dividend price is a martingale, this will be useful latter on. In what follows, we restrict our attention to the case of nil interest rate.

Proposition 2.2 The price at time $t \in[0, T]$ of a credit default swap with spread $\kappa$ is

$$
V_{t}(\kappa)=\mathbb{1}_{\{t<\tau\}} \widetilde{V}_{t}(\kappa), \quad \forall t \in[0, T],
$$

where $\widetilde{V}_{t}(\kappa)$ is a deterministic function associated with the pre-default value of the CDS and equals

$$
\widetilde{V}_{t}(\kappa)=\frac{1}{G(t)}\left(-\int_{t}^{T} \delta(u) d G(u)-\kappa \int_{t}^{T} G(u) d u\right) .
$$

Proof. From Proposition 2.1 we have, on the set $\{t<\tau\}$,

$$
\begin{aligned}
V_{t}(\kappa) & =-\frac{\int_{t}^{T} \delta(u) d G(u)}{G(t)}-\kappa\left(\frac{-\int_{t}^{T} u d G(u)+T G(T)}{G(t)}-t\right) \\
& =\frac{1}{G(t)}\left(-\int_{t}^{T} \delta(u) d G(u)-\kappa\left(T G(T)-t G(t)-\int_{t}^{T} u d G(u)\right)\right) .
\end{aligned}
$$

where, in the last equality, we have used an integration by parts to obtain

$$
\int_{t}^{T} G(u) d u=T G(T)-t G(t)-\int_{t}^{T} u d G(u) .
$$

\footnotetext{
${ }^{1}$ More precisely, the quantity $\delta(\tau)$ is equal to the loss given default associated with the reference entity times the CDS notional amount.
} 


\subsection{Dynamics of CDS Prices in a single default setting}

Here, we compute the dynamics of the CDS's price. It is useful (see 3]) to obtain the hedging strategy of a defaultable claim based on CDS and savings account.

Proposition 2.3 The dynamics of the (ex-dividend) price $V_{t}(\kappa)$ on $[0, T]$ are

$$
d V_{t}(\kappa)=-V_{t-}(\kappa) d M_{t}+\left(1-H_{t}\right)(\kappa-\delta(t) \tilde{\lambda}(t)) d t,
$$

where the $(\mathbb{Q}, \mathbb{H})$-martingale $M$ is given in Proposition 2.1 .

Proof. It suffices to note that

$$
V_{t}(\kappa)=\left(1-H_{t}\right) \widetilde{V}_{t}(\kappa)
$$

with $\widetilde{V}$ given in Proposition 2.2 so that, using integration by parts formula,

$$
d V_{t}(\kappa)=\left(1-H_{t}\right) d \widetilde{V}_{t}(\kappa)-\widetilde{V}_{t-}(\kappa) d H_{t}
$$

Using the explicit expression of $\widetilde{V}_{t}$, we find easily that we have

$$
d \widetilde{V}_{t}(\kappa)=\widetilde{\lambda}(t) \widetilde{V}_{t}(\kappa) d t+(\kappa-\delta(t) \widetilde{\lambda}(t)) d t
$$

The SDE for $V$ follows.

Comment 2.1 It is well known that the risk neutral dynamics of a dividend paying asset is $d S_{t}=d m_{t}-\delta_{t} d t$, where $m$ is a martingale and $\delta$ is the dividend rate. Here, the premium $\kappa$ is similar to a dividend to be paid up to time $t$, hence the quantity $\kappa\left(1-H_{t}\right) d t$ appears. The $\delta(t)$ can be interpreted as a dividend to be received, at time $t$, with probability $\tilde{\lambda}(t) d t$. At default time, the price jumps from $V_{\tau-}$ to 0 , as can be seen in the right-hand side of the dynamics.

Corollary 2.1 The dynamics of the cum-dividend price $V^{\text {cum }}$ on $[0, T]$ are

$$
d V_{t}^{\mathrm{cum}}=\left(\delta(t)-V_{t-}\right) d M_{t} .
$$

Proof. The cumdividend price is

$$
V_{t}^{\text {cum }}=V_{t}+\mathbb{1}_{t<\tau} \delta(\tau)-\kappa(t \wedge \tau)=V_{t}+\int_{0}^{t} \delta(s) d H_{s}-\kappa t\left(1-H_{t}\right)-\int_{0}^{t} \kappa s d H_{s}
$$

THe result follows.

\section{Two default times}

Let us first study the case with two random times $\tau_{1}, \tau_{2}$. For $i=1,2$, we denote by $\left(H_{t}^{i}, t \geq 0\right)$ the default process associated with $\tau_{i}$. The filtration generated by the process $H^{i}$ is denoted $\mathbb{H}^{i}$ and the filtration generated by the two processes $H^{1}, H^{2}$ is $\mathbb{H}=\mathbb{H}^{1} \vee \mathbb{H}^{2}$.

Note that an $\mathcal{H}_{t}^{1} \vee \mathcal{H}_{t}^{2}$-measurable random variable is

- a constant on the set $t<\tau_{1} \wedge \tau_{2}$,

- a $\sigma\left(\tau_{1} \wedge \tau_{2}\right)$-measurable random variable on the set $\tau_{1} \wedge \tau_{2} \leq t<\tau_{1} \vee \tau_{2}$, i.e., a $\sigma\left(\tau_{1}\right)$ measurable random variable on the set $\tau_{1} \leq t<\tau_{2}$, and a $\sigma\left(\tau_{2}\right)$-measurable random variable on the set $\tau_{2} \leq t<\tau_{1}$. We recall that a $\sigma\left(\tau_{1}\right)$-measurable random variable is a Borel function of $\tau_{1}$.

- a $\sigma\left(\tau_{1}, \tau_{2}\right)$-measurable random variable (i.e., a Borel function $h\left(\tau_{1}, \tau_{2}\right)$ ) on the set $\tau_{1} \vee \tau_{2} \leq$ $t$.

To summarize, for fixed $t$, any $\mathcal{H}_{t}^{1} \vee \mathcal{H}_{t}^{2}$-measurable random variable $Z$ admits a representation as

$$
Z=h \mathbb{1}_{t<\tau_{1} \wedge \tau_{2}}+h_{1}\left(\tau_{1}\right) \mathbb{1}_{\tau_{1} \leq t<\tau_{2}}+h_{2}\left(\tau_{2}\right) \mathbb{1}_{\tau_{2} \leq t<\tau_{1}}+h\left(\tau_{1}, \tau_{2}\right) \mathbb{1}_{\tau_{1} \vee \tau_{2} \leq t} .
$$

We denote by $G(t, s)=\mathbb{Q}\left(\tau_{1}>t, \tau_{2}>s\right)$ the survival probability of the pair $\left(\tau_{1}, \tau_{2}\right)$ and we assume that this function is twice differentiable. We denote by $\partial_{i} G$, the partial derivative of $G$ with respect to the $i$-th variable, $i=1,2$ and by $\partial_{1,2} G$, the second order partial derivative of $G$. The density of the pair $\left(\tau_{1}, \tau_{2}\right)$ is denoted by $f$. Simultaneous defaults are precluded in this framework, i.e., $\mathbb{Q}\left(\tau_{1}=\tau_{2}\right)=0$.

Even if the case of two default times is more involved, closed form expressions for the intensities are available. It is important to take into account that the choice of the filtration is very important. Indeed, in general, an $\mathbb{H}^{1}$-martingale is not an $\mathbb{H}^{1} \vee \mathbb{H}^{2}$-martingale. We shall illustrate this important fact below. 


\subsection{Intensities}

We present the computation of martingales associated with default times $\tau_{i}, i=1,2$, in different filtrations. In particular, we shall obtain the computation of the intensities in various filtrations.

\subsubsection{Filtration $\mathbb{H}^{i}$}

We study, for any fixed $i$, the Doob-Meyer decomposition of the sub-martingale $H^{i}$ in the filtration $\mathbb{H}^{i}$. In other terms, we compute the $\mathbb{H}^{i}$-compensator of $H^{i}$. From Proposition 2.1 the process

$$
M_{t}^{(i)}:=H_{t}^{i}-\int_{0}^{t \wedge \tau_{i}} \frac{f_{i}(s)}{G_{i}(s)} d s
$$

is an $\mathbb{H}^{i}$-martingale. Here, $1-G_{i}(s)=F_{i}(s)=\mathbb{Q}\left(\tau_{i} \leq s\right)=\int_{0}^{s} f_{i}(u) d u$. Hence, the process $\left(1-H_{t}^{i}\right) \frac{f_{i}(t)}{G_{i}(t)}$ is the $\mathbb{H}^{i}$-intensity of $\tau^{i}$.

Note that, thanks to Theorem 1.1, any $\mathbb{H}^{i}$-martingale can be written as a stochastic integral with respect to $M^{(i)}$.

\subsubsection{Filtration $\mathbb{H}$}

We recall a result proved in Bielecki et al. 4.

Proposition 3.1 The process $M^{1}$ defined as

$$
M_{t}^{1}:=H_{t}^{1}-\int_{0}^{t \wedge \tau_{1} \wedge \tau_{2}} \frac{\partial_{1} G(s, s)}{G(s, s)} d s-\int_{t \wedge \tau_{1} \wedge \tau_{2}}^{t \wedge \tau_{1}} \frac{\partial_{1,2} G\left(s, \tau_{2}\right)}{\partial_{2} G\left(s, \tau_{2}\right)} d s
$$

is an $\mathbb{H}$-martingale.

Proof. The proof relies on some Itô's calculus to obtain the Doob-Meyer decomposition of $\mathbb{Q}\left(\tau_{1}>t \mid \mathcal{H}_{t}^{2}\right)$. We refer the reader to [4] for details.

This means that the $\mathbb{H}$-intensity of $\tau_{1}$ takes into account the knowledge of $\tau_{2}$ and is equal to the deterministic function $-\frac{\partial_{1} G(t, t)}{G(t, t)}$ on the set $t<\tau_{1} \wedge \tau_{2}$ and to the random quantity $\varphi\left(t, \tau_{2}\right)$ where $\varphi(t, s)=-\frac{\partial_{1,2} G(t, s)}{\partial_{2} G(t, s)}$ on the set $\tau_{2} \leq t<\tau_{1}$.

In a closed form, the processes $H_{t}^{i}-\int_{0}^{t} \lambda_{s}^{i} d s, i=1,2$, are $\mathbb{H}$-martingales, where

$$
\begin{aligned}
\lambda_{t}^{1} & =\left(1-H_{t}^{1}\right)\left(\left(1-H_{t}^{2}\right) \frac{-\partial_{1} G(t, t)}{G(t, t)}-H_{t}^{2} \frac{\partial_{1,2} G\left(t, \tau_{2}\right)}{\partial_{2} G\left(t, \tau_{2}\right)}\right) \\
& =\left(1-H_{t}^{1}\right)\left(1-H_{t}^{2}\right) \widetilde{\lambda}_{t}^{1}+\left(1-H_{t}^{1}\right) H_{t}^{2} \widetilde{\lambda}^{1 \mid 2}\left(\tau_{2}\right), \\
\lambda_{t}^{2} & =\left(1-H_{t}^{2}\right)\left(\left(1-H_{t}^{1}\right) \frac{-\partial_{2} G(t, t)}{G(t, t)}-H_{t}^{1} \frac{\partial_{1,2} G\left(\tau_{1}, t\right)}{\partial_{1} G\left(\tau_{1}, t\right)}\right) \\
& =\left(1-H_{t}^{1}\right)\left(1-H_{t}^{2}\right) \widetilde{\lambda}_{t}^{2}+H_{t}^{1}\left(1-H_{t}^{2}\right) \widetilde{\lambda}_{t}^{2 \mid 1}\left(\tau_{1}\right) .
\end{aligned}
$$

Here

$$
\begin{aligned}
\widetilde{\lambda}_{t}^{i} & =-\frac{\partial_{i} G(t, t)}{G(t, t)} \\
\widetilde{\lambda}_{t}^{1 \mid 2}(s) & =-\frac{\partial_{1,2} G(t, s)}{\partial_{2} G(t, s)}=-\frac{f(t, s)}{\partial_{2} G(t, s)}, \quad \widetilde{\lambda}_{t}^{2 \mid 1}(s)=-\frac{\partial_{1,2} G(s, t)}{\partial_{1} G(s, t)}=-\frac{f(s, t)}{\partial_{1} G(s, t)} .
\end{aligned}
$$

Note that the minus signs in the value of the intensity are due to the fact that $G$ is decreasing with respect to its components, hence the first derivatives are non-positive and the second order derivative $\partial_{1,2} G$ - equal to the density of the pair $\left(\tau_{1}, \tau_{2}\right)$ - is non-negative. The quantity $\widetilde{\lambda}_{t}^{1} d t$ is equal to $\mathbb{Q}\left(\tau_{1} \in d t \mid \tau_{1} \wedge \tau_{2}>t\right)$, that is the probability that $\tau_{1}$ occurs in the time interval $[t, t+d t]$, knowing that neither $\tau_{1}$ nor $\tau_{2}$ have occurred before $t$.

The quantity $\widetilde{\lambda}_{t}^{1 \mid 2}(s)=-\frac{f(t, s)}{\partial_{2} G(t, s)}$ evaluated at $s=\tau_{2}$, represents the value of the default intensity process of $\tau_{1}$ with respect to the filtration $\mathbb{H}$ on the event $\left\{\tau_{2}<t\right\}$. This quantity $\tilde{\lambda}_{t}^{1 \mid 2}(s) d t$ is also the probability that $\tau_{1}$ occurs in the time interval $[t, t+d t]$, knowing that $\tau_{1}$ has not occurred before $t$ and that $s=\tau_{2}$. 
Since we are working in the same filtration 2 the compensated martingale of the counting process $H_{t}=H_{t}^{1}+H_{t}^{2}=\sum_{i=1}^{2} \mathbb{1}_{\tau_{i} \leq t}$ is $M_{t}:=H_{t}-\int_{0}^{t} \lambda_{s} d s$ where

$$
\begin{aligned}
\lambda_{t} & =\lambda_{t}^{1}+\lambda_{t}^{2} \\
& =\left(1-H_{t}^{1}\right)\left(1-H_{t}^{2}\right)\left(-\frac{\partial_{1} G(t, t)+\partial_{2} G(t, t)}{G(t, t)}\right) \\
& -\left(1-H_{t}^{1}\right) H_{t}^{2} \frac{\partial_{1,2} G\left(t, \tau_{2}\right)}{\partial_{2} G\left(t, \tau_{2}\right)}-\left(1-H_{t}^{2}\right) H_{t}^{1} \frac{\partial_{1,2} G\left(\tau_{1}, t\right)}{\partial_{2} G\left(\tau_{1}, t\right)} .
\end{aligned}
$$

\subsection{Dynamics of prices of default contingent claims}

In this section, our aim is to find the dynamics of the price of a contingent claim with payoff $h\left(\tau_{1}, \tau_{2}\right)$. This contains in particular the case of first or second to default claim, with payoff associated with $h(u, v)=\mathbb{1}_{u<v} \varphi(u)$ or $h(u, v)=\mathbb{1}_{u<v<T} \psi(v)$. The goal is to find the dynamics of $Z_{t}:=\mathbb{E}\left(h\left(\tau_{1}, \tau_{2}\right) \mid \mathcal{H}_{t}\right)$.

A first step is to prove

$Z_{t}=h\left(\tau_{1}, \tau_{2}\right) H_{t}^{1} H_{t}^{2}+\psi_{1,0}\left(\tau_{1}, t\right) H_{t}^{1}\left(1-H_{t}^{2}\right)+\psi_{0,1}\left(t, \tau_{2}\right) H_{t}^{2}\left(1-H_{t}^{1}\right)+\left(1-H_{t}^{1}\right)\left(1-H_{t}^{2}\right) \psi_{0,0}(t)$ with

$$
\begin{aligned}
\psi_{1,0}(u, t) & :=\frac{-1}{\partial_{1} G(u, t)} \int_{t}^{\infty} h(u, v) f(u, v) d v \\
\psi_{0,1}(t, v) & :=\frac{-1}{\partial_{2} G(t, v)} \int_{t}^{\infty} h(u, v) f(u, v) d u \\
\psi_{0,0}(t) & :=\frac{1}{G(t, t)} \int_{t}^{\infty} d u \int_{t}^{\infty} d v h(u, v) f(u, v) .
\end{aligned}
$$

The proof follows from iterative conditioning and use of Proposition 2.1. We leave the details to the reader.

One note that, on the one hand, for any function $\phi$,

$$
\begin{aligned}
H_{t}^{1} H_{t}^{2} \phi\left(\tau_{1}, \tau_{2}\right) & =\int_{0}^{t} d H_{u}^{1} \int_{0}^{t} d H_{v}^{2} \phi(u, v), \\
H_{t}^{1} \phi\left(\tau_{1}, t\right) & =\int_{0}^{t} \phi(u, t) d H_{u}^{1}
\end{aligned}
$$

so that, using integration by parts formula and re-arranging the terms

$$
\begin{aligned}
d Z_{t}= & \left(\left(h\left(t, \tau_{2}\right)-\psi_{0,1}\left(t, \tau_{2}\right)\right) H_{t}^{2}+\left(\psi_{1,0}(t, t)-\psi_{0,0}(t)\right)\left(1-H_{t}^{2}\right)\right) d H_{t}^{1} \\
+ & \left(\left(h\left(\tau_{1}, t\right)-\psi_{1,0}\left(\tau_{1}, t\right)\right) H_{t}^{1}+\left(\psi_{0,1}(t, t)-\psi_{0,0}(t)\right)\left(1-H_{t}^{1}\right)\right) d H_{t}^{2} \\
+ & \left(\left(1-H_{t}^{2}\right) \int_{0}^{t} \partial_{2} \psi_{1,0}(u, t) d H_{u}^{1}+\left(1-H_{t}^{1}\right) \int_{0}^{t} \partial_{1} \psi_{0,1}(t, v) d H_{v}^{2}\right. \\
& \left.+\left(1-H_{t}^{1}\right)\left(1-H_{t}^{2}\right) \frac{d}{d t} \psi_{0,0}(t)\right) d t
\end{aligned}
$$

On the other hand, one checks that, with easy computation, that

$$
\begin{aligned}
\partial_{1} \psi_{0,1}(t, v) & =\lambda^{1 \mid 2}(t, v)\left(\psi_{0,1}(t, v)-h(t, v)\right), \\
\left.\partial_{2} \psi_{1,0}(u, t)\right) & =\lambda^{2 \mid 1}(u, t)\left(\psi_{1,0}(u, t)-h(u, t)\right), \\
\frac{d}{d t} \psi_{0,0}(t) & =\left(\widetilde{\lambda}_{1}(t)+\widetilde{\lambda}_{2}(t)\right) \psi_{0,0}(t)+\frac{1}{G(t, t)}\left(\partial_{1} G(t, t) \psi_{1,0}(t, t)+\partial_{2} G(t, t) \psi_{0,1}(t, t)\right) .
\end{aligned}
$$

It follows that

$$
\begin{aligned}
d Z_{t}= & \left(\left(h\left(t, \tau_{2}\right)-\psi_{0,1}\left(t, \tau_{2}\right)\right) H_{t}^{2}+\left(\psi_{1,0}(t, t)-\psi_{0,0}(t)\right)\left(1-H_{t}^{2}\right)\right) d M_{t}^{1} \\
& +\left(\left(h\left(\tau_{1}, t\right)-\psi_{1,0}\left(\tau_{1}, t\right)\right) H_{t}^{1}+\left(\psi_{0,1}(t, t)-\psi_{0,0}(t)\right)\left(1-H_{t}^{1}\right)\right) d M_{t}^{2}
\end{aligned}
$$

${ }^{2}$ The sum of two martingales in the same filtration is a martingale. 


\subsection{Dynamics of CDS prices}

Let us now examine the valuation of a single-name CDS written on the default $\tau_{1}$. Our aim is to show that the dynamics of this CDS will be affected by the information on $\tau_{2}$ : when $\tau_{2}$ occurs, the intensity of $\tau_{1}$ changes, and this will change the parameters of the price dynamics. We reproduce some results appearing in Bielecki et al. 4].

We consider a CDS

- with a constant $\operatorname{spread} \kappa$

- which delivers $\delta\left(\tau_{1}\right)$ at time $\tau_{1}$ if $\tau_{1}<T$, where $\delta$ is a deterministic function.

The value of the CDS takes the form

$$
V_{t}(\kappa)=\widetilde{V}_{t}(\kappa) \mathbb{1}_{t<\tau_{1} \wedge \tau_{2}}+\widehat{V}_{t}(\kappa) \mathbb{1}_{\tau_{1} \wedge \tau_{2} \leq t<\tau_{1}} .
$$

First, we restrict our attention to the case $t<\tau_{1} \wedge \tau_{2}$.

Proposition 3.2 On the set $\left\{t<\tau_{1} \wedge \tau_{2}\right\}$, the value of the CDS is

$$
\widetilde{V}_{t}(\kappa)=\frac{1}{G(t, t)}\left(-\int_{t}^{T} \delta(u) \partial_{1} G(u, t) d u-\kappa \int_{t}^{T} G(u, t) d u\right) .
$$

Proof. The value $V(\kappa)$ of this CDS, computed in the filtration $\mathbb{H}$, i.e., taking care on the information on the second default contained in that filtration, is

$$
V_{t}(\kappa)=\mathbb{1}_{t<\tau_{1}} \mathbb{E}\left(\delta\left(\tau_{1}\right) \mathbb{1}_{\tau_{1} \leq T}-\kappa\left(\left(T \wedge \tau_{1}\right)-t\right) \mid \mathcal{H}_{t}\right)
$$

Let us denote by $\tau=\tau_{1} \wedge \tau_{2}$ the first default time. Then, $\mathbb{1}_{\{t<\tau\}} V_{t}(\kappa)=\mathbb{1}_{\{t<\tau\}} \widetilde{V}_{t}(\kappa)$, where

$$
\begin{aligned}
\widetilde{V}_{t}(\kappa)= & \frac{1}{\mathbb{Q}(\tau>t)} \mathbb{E}\left(\delta\left(\tau_{1}\right) \mathbb{1}_{\tau_{1} \leq T} \mathbb{1}_{t<\tau}-\kappa\left(\left(T \wedge \tau_{1}\right)-t\right) \mathbb{1}_{t<\tau}\right) \\
= & \frac{1}{G(t, t)} \mathbb{E}\left(\delta\left(\tau_{1}\right) \mathbb{1}_{\tau_{1} \leq T} \mathbb{1}_{t<\tau}-\kappa\left(\left(T \wedge \tau_{1}\right)-t\right) \mathbb{1}_{t<\tau}\right) \\
= & \frac{1}{G(t, t)}\left(\int_{t}^{T} \delta(u) \mathbb{Q}\left(\tau_{1} \in d u, \tau_{2}>t\right)\right. \\
& \left.\quad-\kappa \int_{t}^{T}(u-t) \mathbb{Q}\left(\tau_{1} \in d u, \tau_{2}>t\right)-(T-t) \kappa \int_{T}^{\infty} \mathbb{Q}\left(\tau_{1} \in d u, \tau_{2}>t\right)\right) .
\end{aligned}
$$

In other terms, using integration by parts formula, we end up with

$$
\widetilde{V}_{t}(\kappa)=\frac{1}{G(t, t)}\left(-\int_{t}^{T} \delta(u) \partial_{1} G(u, t) d u-\kappa \int_{t}^{T} G(u, t) d u\right) .
$$

Proposition 3.3 On the event $\left\{\tau_{2} \leq t<\tau_{1}\right\}$, the CDS price equals

$$
\begin{aligned}
V_{t}(\kappa) & =\widehat{V}_{t}(\kappa)=\mathbb{E}\left(\delta\left(\tau_{1}\right) \mathbb{1}_{\tau_{1} \leq T}-\kappa\left(\left(T \wedge \tau_{1}\right)-t\right) \mid \sigma\left(\tau_{2}\right)\right) \\
& =\frac{1}{\partial_{2} G\left(t, \tau_{2}\right)}\left(-\int_{t}^{T} \delta(u) f\left(u, \tau_{2}\right) d u-\kappa \int_{t}^{T} \partial_{2} G\left(u, \tau_{2}\right) d u\right):=V_{t}^{1 \mid 2}\left(\tau_{2}\right)
\end{aligned}
$$

where

$$
V_{t}^{1 \mid 2}(s)=\frac{1}{\partial_{2} G(t, s)}\left(-\int_{t}^{T} \delta(u) f(u, s) d u-\kappa \int_{t}^{T} \partial_{2} G(u, s) d u\right) .
$$

In the financial interpretation, $V_{t}^{1 \mid 2}(s)$ is the market price at time $t$ of a CDS on the first credit name, under the assumption that the default $\tau_{2}$ occurs at time $s$ and the first name has not yet defaulted (recall that simultaneous defaults are excluded, since we have assumed that $G$ is differentiable).

The price of a CDS is $V_{t}=\widetilde{V}_{t} \mathbb{1}_{t<\tau_{2} \wedge \tau_{1}}+\widehat{V}_{t} \mathbb{1}_{\tau_{2} \wedge \tau_{1} \leq t<\tau_{1}}$. Differentiating the deterministic function which gives the value of the CDS, we obtain

$$
d \widetilde{V}_{t}(\kappa)=\left(\left(\widetilde{\lambda}_{1}(t)+\tilde{\lambda}_{2}(t)\right) \widetilde{V}_{t}(\kappa)+\kappa-\widetilde{\lambda}_{1}(t) \delta(t)-\tilde{\lambda}_{2}(t) V_{t}^{1 \mid 2}(t)\right) d t
$$

where for $i=1,2$ the function $\widetilde{\lambda}_{i}(t)$ is the (deterministic) pre-default intensity of $\tau_{i}$ given in (4) and

$$
d \widehat{V}_{t}(\kappa)=\left(\widetilde{\lambda}_{t}^{1 \mid 2}\left(\tau_{2}\right)\left(\widehat{V}_{t}(\kappa)-\delta(t)\right)+\kappa\right) d t
$$

where $\widetilde{\lambda}_{t}^{1 \mid 2}(u)$ is given in $(5)$. 
Proposition 3.4 The price of a CDS follows

$$
\begin{aligned}
d V_{t}= & \left(1-H_{t}^{1}\right)\left(1-H_{t}^{2}\right)\left(\kappa-\delta(t) \widetilde{\lambda}^{1}(t)\right) d t+\left(1-H_{t}^{1}\right) H_{t}^{2}\left(\kappa-\delta(t) \widetilde{\lambda}_{t}^{1 \mid 2}\left(\tau_{2}\right)\right) d t \\
& -V_{t-} d M_{t}^{1}+\left(1-H_{t}^{1}\right)\left(V_{t}^{1 \mid 2}(t)-V_{t-}\right) d M_{t}^{2} .
\end{aligned}
$$

Proof. Differentiating $V_{t}=\widetilde{V}_{t}\left(1-H_{t}^{1}\right)\left(1-H_{t}^{2}\right)+\widehat{V}_{t}\left(1-H_{t}^{1}\right) H_{t}^{2}$ one obtains

$$
\begin{aligned}
d V_{t}= & \left(1-H_{t}^{1}\right)\left(1-H_{t}^{2}\right) d \widetilde{V}_{t}+\left(1-H_{t}^{1}\right) H_{t}^{2} d \widehat{V}_{t}-V_{t-} d H_{t}^{1} \\
& +\left(1-H_{t}^{1}\right)\left(V_{t}^{1 \mid 2}(t)-\widetilde{V}_{t}\right) d H_{t}^{2},
\end{aligned}
$$

which leads to the result after light computations.

Comment 3.1 As for a single name CDS, the quantity $-\delta(t) \widetilde{\lambda}^{1}(t)$ corresponds to the dividend $\delta$ to be paid at time $t$ with probability $\widetilde{\lambda}^{1}(t) d t$ on the set $t<\tau_{1} \wedge \tau_{2}$ and $-\delta(t) \widetilde{\lambda}_{t}^{1 / 2}$ corresponds to the dividend $\delta$ to be paid at time $t$ with probability $\widetilde{\lambda}_{t}^{1 \mid 2} d t$ on the set $\tau_{2}<t<\tau_{1}$. The quantity $V_{t}^{1 \mid 2}(t)-\widetilde{V}_{t}$ represents the jump in the value of the CDS, when default $\tau_{2}$ occurs at time $t$.

The cumulative price of the CDS is

$$
V_{t}^{\text {cum }}=\mathbb{E}\left(\delta\left(\tau_{1}\right) \mathbb{1}_{\tau_{1} \leq T}-\kappa\left(T \wedge \tau_{1}\right) \mid \mathcal{H}_{t}\right)
$$

It follows that

$$
d V_{t}^{\mathrm{cum}}=d V_{t}+\delta(t) d H_{t}^{1}-\kappa\left(1-H_{t}^{1}\right) d t
$$

hence, since the cumulative price is a martingale

$$
d V_{t}=d m_{t}-\delta(t) \lambda_{t}^{1} d t+\kappa\left(1-H_{t}^{1}\right) d t
$$

where $d m_{t}=d V_{t}^{\text {cum }}-\delta(t) d M_{t}^{1}$. This is an easy way to obtain the drift term in Equation [6]. It turns out that the cum-dividend CDS price process has the following dynamics

$$
\begin{aligned}
d V_{t}^{\text {cum }}= & \left(1-H_{t}^{1}\right)\left(1-H_{t}^{2}\right)\left(\kappa-\delta(t) \widetilde{\lambda}^{1}(t)\right) d t+\left(1-H_{t}^{1}\right) H_{t}^{2}\left(\kappa-\delta(t) \widetilde{\lambda}_{t}^{1 \mid 2}\right) d t \\
& -V_{t-} d M_{t}^{1}+\left(1-H_{t}^{1}\right)\left(V_{t}^{1 \mid 2}(t)-V_{t-}\right) d M_{t}^{2}+\delta(t) d H_{t}^{1}-\kappa\left(1-H_{t}^{1}\right) d t \\
= & \left(\delta(t)-V_{t-}\right) d M_{t}^{1}+\left(1-H_{t}^{1}\right)\left(V_{t}^{1 \mid 2}(t)-V_{t-}\right) d M_{t}^{2} .
\end{aligned}
$$

\subsection{CDSs as hedging assets}

Assume now that a CDS written on $\tau_{2}$ is also traded in the market. We denote by $\delta^{i}, i=1,2$ the recovery (assumes to be deterministic) and $V^{i}, i=1,2$ the prices of the two CDSs. Since the CDS are paying dividends, a self financing strategy consisting in $\vartheta^{i}$ shares of CDS's has value $X_{t}=\vartheta_{t}^{1} V_{t}^{1}+\vartheta_{t}^{2} V_{t}^{2}$ and dynamics

$$
\begin{aligned}
& d X_{t}=\vartheta_{t}^{1} d V_{t}^{1, \text { cum }}+\vartheta_{t}^{2} d V_{t}^{2, \text { cum }} \\
& =\vartheta_{t}^{1}\left(\left(\delta^{1}(t)-V_{t-}^{1}\right) d M_{t}^{1}+\left(1-H_{t}^{1}\right)\left(V_{t}^{1 \mid 2}(t)-\widetilde{V}_{t}^{1}\right) d M_{t}^{2}\right) \\
& +\vartheta_{t}^{2}\left(\left(\delta^{2}(t)-V_{t-}^{2}\right) d M_{t}^{2}+\left(1-H_{t}^{2}\right)\left(V_{t}^{2 \mid 1}(t)-\widetilde{V}_{t}^{2}\right) d M_{t}^{1}\right) \\
& =\left(\vartheta_{t}^{1}\left(\delta^{1}(t)-V_{t-}^{1}\right)+\vartheta_{t}^{2}\left(1-H_{t}^{2}\right)\left(V_{t}^{2 \mid 1}(t)-\widetilde{V}_{t}^{2}\right) d M_{t}^{1}\right. \\
& +\left(\vartheta_{t}^{1}\left(1-H_{t}^{1}\right)\left(V_{t}^{1 \mid 2}(t)-\widetilde{V}_{t}^{1}\right)+\vartheta_{t}^{2}\left(\delta^{2}(t)-V_{t-}^{2}\right)\right) d M_{t}^{2}
\end{aligned}
$$

Let $A \in \mathcal{H}_{T}$ be a terminal payoff with price $A_{t}=\mathbb{E}\left(A \mid \mathcal{H}_{t}\right)$, then from Theorem 1.1 there exists predictable processes $\pi^{1}$ and $\pi^{2}$ such that

$$
A_{t}=\mathbb{E}(A)+\int_{0}^{t} \pi_{s}^{1} d M_{s}^{1}+\int_{0}^{t} \pi_{s}^{2} d M_{s}^{2} .
$$

In order to hedge that claim, it remains to solve the linear system

$$
\begin{aligned}
& \vartheta_{t}^{1}\left(\delta^{1}(t)-V_{t-}^{1}\right)+\vartheta_{t}^{2}\left(1-H_{t}^{2}\right)\left(V_{t}^{2 \mid 1}(t)-\widetilde{V}_{t}^{2}\right)=\pi_{t}^{1} \\
& \vartheta_{t}^{1}\left(1-H_{t}^{1}\right)\left(V_{t}^{1 \mid 2}(t)-\widetilde{V}_{t}^{1}\right)+\vartheta_{t}^{2}\left(\delta^{2}(t)-V_{t-}^{2}\right)=\pi_{t}^{2}
\end{aligned}
$$


Hence, on the set $t<\tau_{1} \wedge \tau_{2}$, noting that $V_{t}^{i}=\widetilde{V}_{t}^{i}$ on that set,

$$
\begin{aligned}
\vartheta_{t}^{1} & =\frac{\pi_{t}^{1}\left(\delta^{2}(t)-\widetilde{V}_{t}^{2}\right)-\pi_{t}^{2}\left(V_{t}^{2 \mid 1}(t)-\widetilde{V}_{t}^{2}\right)}{\left(\delta^{1}(t)-\widetilde{V}_{t}^{1}\right)\left(\delta^{2}(t)-\widetilde{V}_{t}^{2}\right)-\left(V_{t}^{1 \mid 2}-\widetilde{V}_{t}^{1}\right)\left(V_{t}^{2 \mid 1}-\widetilde{V}_{t}^{2}\right)}, \\
\vartheta_{t}^{2} & =\frac{\pi_{t}^{2}\left(\delta^{1}(t)-\widetilde{V}_{t}^{1}\right)-\pi_{t}^{1}\left(V_{t}^{1 \mid 2}-\widetilde{V}_{t}^{1}\right)}{\left(\delta^{1}(t)-\widetilde{V}_{t}^{1}\right)\left(\delta^{2}(t)-\widetilde{V}_{t}^{2}\right)-\left(V_{t}^{1 \mid 2}-\widetilde{V}_{t}^{1}\right)\left(V_{t}^{2 \mid 1}(t)-\widetilde{V}_{t}^{2}\right)} .
\end{aligned}
$$

On the set $\tau_{1}<t<\tau_{2}$

$$
\vartheta_{t}^{1}=\frac{\pi_{t}^{1}\left(\delta^{2}(t)-V_{t}^{2}\right)-\pi_{t}^{2}\left(V_{t}^{2 \mid 1}-V_{t}^{2}\right)}{\left(\delta^{1}(t)-V_{t}^{1}\right)\left(\delta^{2}(t)-V_{t}^{2}\right)}, \quad \vartheta_{t}^{2}=\frac{\pi_{t}^{2}}{\delta^{2}(t)-V_{t}^{2}} .
$$

On the set $\tau_{2}<t<\tau_{1}$

$$
\vartheta_{t}^{1}=-\frac{\pi_{t}^{1}}{\delta^{1}(t)-V_{t}^{1}}, \quad \vartheta_{t}^{2}=\frac{\pi_{t}^{2}\left(\delta^{1}(t)-V_{t}^{1}\right)-\pi_{t}^{1}\left(V_{t}^{1 \mid 2}-V_{t}^{2}\right)}{\left(\delta^{1}(t)-V_{t}^{1}\right)\left(\delta^{2}(t)-V_{t}^{2}\right)} .
$$

On the set $\tau_{1} \vee \tau_{2}<t$

$$
\vartheta_{t}^{1}=\frac{\pi_{t}^{1}}{\delta^{1}(t)-V_{t}^{1}}, \quad \vartheta_{t}^{2}=\frac{\pi_{t}^{2}}{\delta^{2}(t)-V_{t}^{2}} .
$$

As we saw above, for the case $A=h\left(\tau_{1}, \tau_{2}\right)$, one has a closed form for the coefficients $\pi$ :

$$
\begin{aligned}
& \pi_{t}^{1}=\left(h\left(t, \tau_{2}\right)-\psi_{0,1}\left(t, \tau_{2}\right)\right) H_{t}^{2}+\left(\psi_{1,0}(t, t)-\psi_{0,0}(t)\right)\left(1-H_{t}^{2}\right) \\
& \pi_{t}^{2}=\left(h\left(\tau_{1}, t\right)-\psi_{1,0}\left(\tau_{1}, t\right)\right) H_{t}^{1}+\left(\psi_{0,1}(t, t)-\psi_{0,0}(t)\right)\left(1-H_{t}^{1}\right)
\end{aligned}
$$

\subsection{Multidefault setting}

The same methodology can be applied in a multidefault setting. The only difficulty is that one has to rank the defaults and to distinguish the various possibilities. For example, for three defaults, the dynamics of the cumdividend price of the CDS written on the default $\tau_{1}$ is

$$
\begin{aligned}
d V_{t}^{\text {cum }}= & \left(\delta^{1}(t)-V_{t-}\right) d M_{t}^{1}+\left(1-H_{t}^{1}\right)\left(\left(1-H_{t}^{3}\right) V_{t}^{1 \mid 2}(t)+H_{t}^{3} V_{t}^{1 \mid 32}\left(t, \tau_{3}\right)-V_{t-}\right) d M_{t}^{2} \\
& +\left(1-H_{t}^{1}\right)\left(\left(1-H_{t}^{2}\right) V_{t}^{1 \mid 3}(t)+H_{t}^{2} V_{t}^{1 \mid 23}\left(\tau_{2}, t\right)-V_{t-}\right) d M_{t}^{3},
\end{aligned}
$$

where $V_{t}$ is the (ex-dividend) price of the CDS at time $t, V_{t}^{1 \mid 2}(u)$ is the price of the CDS on the set $\left\{\tau_{2}=u\right\}$ for $u<t<\tau_{3}, V_{t}^{1 \mid 3}(v)$ is the price of the CDS on the set $\left\{\tau_{3}=v\right\}$ for $v<t<\tau_{2}$, $V_{t}^{1 \mid 23}(u, v)$ is the price of the CDS, on the set $\left\{\tau_{2}=u, \tau_{3}=v\right\}$ for $u<v<t$ and $V_{t}^{1 \mid 32}(u, v)$ is the price of the CDS, on the set $\left\{\tau_{2}=u, \tau_{3}=v\right\}$ for $v<u<t$.

\section{Particular case: ordered defaults}

In this section, we consider the particular case where default times are ordered. In this situation, single-name CDS can be viewed as $k$ th-to-default swaps. We first consider a setting with two names only, then we investigate the hedging of loss derivatives written on a multivariate $n$ dimensional credit portfolio.

\subsection{Dynamics of CDS prices in a two defaults setting}

Let us now assume that $\tau_{1}<\tau_{2}$, a.s. In that case, $G(t, s)=G(t, t)$ for $s \leq t$, hence the martingale $M^{1}$ defined in Proposition 3.1 simplifies:

$$
M_{t}^{1}=H_{t}^{1}-\int_{0}^{t \wedge \tau_{1}} \frac{\partial_{1} G(s, s)}{G(s, s)} d s=H_{t}^{1}-\int_{0}^{t \wedge \tau_{1}} \frac{f_{1}(s)}{G_{1}(s)} d s
$$

where

$$
G_{1}(s)=\mathbb{Q}\left(\tau_{1}>s\right)=G(s, s)=\int_{s}^{\infty} f_{1}(u) d u=\int_{s}^{\infty} \partial_{1} G(u, u) d u
$$


The process $M^{1}$ is $\mathbb{H}^{1}$-adapted, hence is an $\mathbb{H}^{1}$-martingale and it follows from Theorem 1.1 that any $\mathbb{H}^{1}$-martingale is a $\mathbb{H}$-martingale. Furthermore, the intensity of $\tau_{2}$ vanishes on the set $t<\tau_{1}$ and

$$
M_{t}^{2}=H_{t}^{2}-\int_{t \vee \tau_{1}}^{t \wedge \tau_{2}} \frac{f\left(\tau_{1}, s\right)}{\partial_{1} G\left(\tau_{1}, s\right)} d s=H_{t}^{2}-\int_{0}^{t} \mathbb{1}_{\tau_{1}<s<\tau_{2}} \frac{f\left(\tau_{1}, s\right)}{\partial_{1} G\left(\tau_{1}, s\right)} d s
$$

Proposition 4.1 Let $V^{i}, i=1,2$ be the price of a CDS on $\tau_{i}$, with contractual spread $\kappa_{i}$ and payment at default given by a deterministic function $\delta_{i}$. The $\mathbb{H}$-dynamics of $V^{1}$ is

$$
d V_{t}^{1}=-V_{t-}^{1} d M_{t}^{1}+\left(1-H_{t}^{1}\right)\left(\kappa_{1}-\delta_{1}(t) \widetilde{\lambda}_{1}(t)\right) d t
$$

with $\widetilde{\lambda}_{1}(t)=\frac{f_{1}(t)}{G_{1}(t)}$. The $\mathbb{H}$-dynamics of $V^{2}$ is

$$
d V_{t}^{2}=-V_{t-}^{2} d M_{t}^{2}+\left(1-H_{t}^{2}\right) \kappa_{2} d t-\left(1-H_{t}^{2}\right) H_{t}^{1} \delta_{2}(t) \widetilde{\lambda}_{t}^{2 \mid 1}\left(\tau_{1}\right) d t+\left(V_{t}^{2 \mid 1}(t)-V_{t-}^{2}\right) d M_{t}^{1}
$$

Proof. Apply Proposition 2.3 or (6) to obtain (7), and (6) to obtain (8).

\subsection{Multidefault setting}

Let $G$ be the survival function of the joint defaults, assumed to be differentiable

$$
G\left(t_{1}, \ldots, t_{n}\right)=\mathbb{Q}\left(\tau_{1}>t_{1}, \ldots, \tau_{n}>t_{n}\right)
$$

and

$$
G_{j}\left(t_{1}, \ldots, t_{j}\right)=\mathbb{Q}\left(\tau_{1}>t_{1}, \ldots, \tau_{j}>t_{j}\right) .
$$

We shall denote by $f$ the density of the $n$-uple $\left(\tau_{i}, i \leq n\right)$ and by $f_{j}$ the density of the $j$-uple $\left(\tau_{i}, i \leq j\right)$. Since the defaults are ordered, setting $t_{j}^{1}=t_{1}, \ldots, t_{j}$ one has

$$
\begin{aligned}
G_{j}\left(t_{j}^{1}\right) & :=\mathbb{Q}\left(\tau_{1}>t_{1}, \ldots, \tau_{j}>t_{j}\right) \\
& =\mathbb{Q}\left(\tau_{1}>t_{1}, \ldots, \tau_{j}>t_{j}, \tau_{j+1}>t_{j}, \ldots, \tau_{n}>t_{j}\right)=G\left(t_{j}^{1}, t_{j}, \ldots, t_{j}\right)
\end{aligned}
$$

The fundamental martingales are

$$
M_{t}^{j}=H_{t}^{j}-\int_{t \vee \tau_{j-1}}^{t \wedge \tau_{j}} \tilde{\lambda}_{s}^{j \mid j-1}\left(\tau_{1}, \ldots, \tau_{j-1}\right) d s
$$

where

$$
\tilde{\lambda}_{t}^{j \mid j-1}\left(t_{j-1}^{1}\right)=-\frac{\partial_{1, j} G_{j}\left(t_{j-1}^{1}, t\right)}{\partial_{1, j-1} G_{j}\left(t_{j-1}^{1}, t\right)}
$$

and $\partial_{1, j}=\partial_{1} \ldots \partial_{j}$.

Proposition 4.2 If $V^{i}$ is the price process of a CDS with maturity $T$, written on the $i$-th default, with spread $\kappa_{i}$ and payment at default given by a deterministic function $\delta_{i}$, then

$$
\begin{aligned}
d V_{t}^{i}= & -V_{t-}^{i} d M_{t}^{i}-\left(1-H_{t}^{i}\right) H_{t}^{i-1} \delta_{i}(t) \widetilde{\lambda}_{t}^{i \mid i-1}\left(\tau_{1}, \ldots, \tau_{i-1}\right) d t \\
& +\sum_{j=1}^{i-1}\left(V_{t}^{i \mid j}\left(\tau_{1}, \ldots, \tau_{j}\right)-V_{t-}^{i}\right) d M_{t}^{j}+\left(1-H_{t}^{i}\right) \kappa_{i} d t
\end{aligned}
$$

where

$$
V_{t}^{i \mid j}\left(t_{j}^{1}\right)=\frac{-\int_{t}^{T} \delta_{i}(u) f_{j+1}\left(t_{j}^{1}, u\right) d u-\kappa_{i} \int_{t}^{T} \partial_{1, j} G_{j+1}\left(t_{j}^{1}, u\right) d u}{\partial_{1, j} G_{j}\left(t_{j}^{1}\right)} .
$$

\subsection{Hedging of a loss}

In order to hedge the payoff $B$, one proceeds in two steps. The first step is to compute the martingale representation of $\mathbb{E}\left(B \mid \mathcal{H}_{t}\right)$, i.e., identify the predictable processes $\pi$ such that

$$
\mathbb{E}\left(B \mid \mathcal{H}_{t}\right)=\mathbb{E}(B)+\sum_{j=1}^{n} \int_{0}^{t} \pi_{s}^{j} d M_{s}^{j}
$$


We denote by $D^{i}$ the dividend part associated with the CDS written on $\tau_{i}$. A self-financing strategy with value

$$
V_{t}=\vartheta_{t}^{0}+\sum_{i=1}^{n} \vartheta_{t}^{i} V_{t}^{i}
$$

satisfies

$$
\begin{aligned}
d V_{t} & =\sum_{i=1}^{n} \vartheta_{t}^{i}\left(d V_{t}^{i}+d D_{t}^{i}\right)=\sum_{i=1}^{n} \vartheta_{t}^{i}\left(\left(\delta^{i}(t)-V_{t-}^{i}\right) d M_{t}^{i}+\sum_{j=1}^{i-1}\left(V_{t}^{i \mid j}-V_{t-}^{i}\right) d M_{t}^{j}\right) \\
& =\sum_{j=1}^{n} d M_{t}^{j} \sum_{i=j}^{n} \vartheta_{t}^{i}\left(V_{t}^{i \mid j}-V_{t-}^{i}\right)
\end{aligned}
$$

where we set $V_{t}^{i \mid i}=\delta^{i}(t)$. It remains to solve the linear system (with unknown $\vartheta$ )

$$
\sum_{i=j}^{n} \vartheta_{t}^{i}\left(V_{t}^{i \mid j}-V_{t-}^{i}\right)=\pi_{t}^{j}, j=1, \ldots, n .
$$

As an example, we now compute the conditional law of the loss, i.e., $\mathbb{E}\left(f\left(L_{T}\right) \mid \mathcal{H}_{t}\right)$ where $L_{T}=\sum_{k=1}^{n} \mathbb{1}_{\tau_{k} \leq T}$. Let $B_{t}^{k}(T)=\mathbb{E}\left(\mathbb{1}_{T<\tau_{k}} \mid \mathcal{H}_{t}\right)$ (or simply $B_{t}^{k}$ ) be the price of a defaultable zero-coupon written on the $k$-th default, with maturity $T$.

$$
\mathbb{E}\left(f\left(L_{T}\right) \mid \mathcal{H}_{t}\right)=\sum_{k=1}^{n} f(k)\left(B_{t}^{k+1}-B_{t}^{k}\right)=f(n)+\sum_{k=1}^{n} B_{t}^{k}(f(k-1)-f(k))
$$

Obviously, setting $\tau_{0}=0$

$$
B_{t}^{k}=\sum_{j=1}^{k} \mathbb{1}_{\tau_{j-1} \leq t<\tau_{j}} \mathbb{E}\left(\mathbb{1}_{T<\tau_{k}} \mid \mathcal{H}_{t}\right)=\sum_{j=1}^{k} \mathbb{1}_{\tau_{j-1} \leq t<\tau_{j}} \frac{\mathbb{Q}\left(T<\tau_{k}, t<\tau_{j} \mid \mathcal{H}_{t}^{j-1}\right)}{\mathbb{Q}\left(t<\tau_{j} \mid \mathcal{H}_{t}^{j-1}\right)}
$$

Now, on the set $\tau_{j-1} \leq t$

$$
\mathbb{Q}\left(T<\tau_{k}, t<\tau_{j} \mid \mathcal{H}_{t}^{j-1}\right)=\Phi_{k, j-1}\left(\tau_{1}, \ldots, \tau_{j-1}, t, T\right)
$$

where, for $j \leq k-1$

$$
\begin{aligned}
\Phi_{k, j-1}\left(t_{1}, \ldots, t_{j-1}, t, T\right) & =\frac{\mathbb{Q}\left(t<\tau_{j}, T<\tau_{k}, \tau_{1} \in d t_{1}, \ldots, \tau_{j-1} \in d t_{j-1}\right)}{\mathbb{Q}\left(\tau_{1} \in d t_{1}, \ldots, \tau_{j-1} \in d t_{j-1}\right)} \\
& =\frac{\partial_{1, j-1} G_{k}\left(t_{j-1}^{1}, t, \ldots, t, T\right)}{\partial_{1, j-1} G_{k}\left(t_{j-1}^{1}, \ldots, t_{j-1}\right)}
\end{aligned}
$$

On the set $\tau_{k-1} \leq t($ for $j=k$ )

$$
\frac{\mathbb{Q}\left(T<\tau_{k}, t<\tau_{k} \mid \mathcal{H}_{t}^{k-1}\right)}{\mathbb{Q}\left(t<\tau_{k} \mid \mathcal{H}_{t}^{k-1}\right)}=\frac{\mathbb{Q}\left(T<\tau_{k} \mid \mathcal{H}_{t}^{k-1}\right)}{\mathbb{Q}\left(t<\tau_{k} \mid \mathcal{H}_{t}^{k-1}\right)}=\frac{\partial_{1, k-1} G_{k}\left(t_{k-1}^{1}, T\right)}{\partial_{1, k-1} G_{k}\left(t_{k-1}^{1}, t\right)} .
$$

Since

$$
d B_{t}^{k}=-B_{t-}^{k} d M_{t}^{k}+\sum_{j=1}^{k-1} \nu_{t}^{j, k} d M_{t}^{j}=\sum_{j=1}^{k} \nu_{t}^{j, k} d M_{t}^{j}
$$

where, for $j<k$, we have set $\nu_{t}^{j, k}=\frac{\Phi_{k, j}\left(\tau_{1}, \ldots, \tau_{j-1}, t, t, T\right)}{\Phi_{k, j}\left(\tau_{1}, \ldots, \tau_{j-1}, t, t, t\right)}-\frac{\Phi_{k, j-1}\left(\tau_{1}, \ldots, \tau_{j-1}, t, T\right)}{\Phi_{k, j-1}\left(\tau_{1}, \ldots, \tau_{j-1}, t, t\right)}$ and $\nu_{t}^{k, k}=-B_{t-}^{k}$. It follows that, setting $X_{t}=\mathbb{E}\left(f\left(L_{T}\right) \mid \mathcal{H}_{t}\right)$,

$$
d X_{t}=\sum_{j=1}^{n} d M_{t}^{j} \sum_{k=j}^{n}(f(k-1)-f(k)) \nu_{t}^{i, k}
$$

and the hedging strategy for the contingent claim $f\left(L_{T}\right)$ is the solution $\vartheta$ of the triangular system

$$
\sum_{i=j}^{n} \vartheta_{t}^{i}\left(V_{t}^{i \mid j}-V_{t-}^{i}\right)=\sum_{k=j}^{n}(f(k-1)-f(k)) \nu_{t}^{j, k}, j=1, \ldots, n .
$$




\section{Conclusion}

We investigate a quite general pure jump setting where the density of joint default is known. We compute the default intensities in the filtration of all the default times. In particular, at each instant when a default event occurs, default intensities of non-defaulted names are dynamically updated. This leads to a dependence structure among default times which is regularly updated as defaults arrive. We have seen that the hedging of loss derivatives such as CDO tranches or basket default swaps can be fully described in this framework with no Markovian assumption. The hedging strategies with respect to single-name CDS can be derived analytically in a twodefaults setting. Even if similar ideas can be exploited in higher dimension, the construction of dynamic hedging strategies would involve very cumbersome computations if one wants to

consider all possible default scenarios. Interestingly, in the particular case of ranked default times, the hedging issue can be solved explicitly.

\section{References}

[1] Basel Committee on Banking Supervision (2009), Guidelines for computing capital for incremental risk in the trading book.

[2] Bielecki, T.R. and Rutkowski, M. (2001), Credit risk: Modelling Valuation and Hedging, Springer Verlag, Berlin.

[3] Bielecki, T.R., Jeanblanc, M., and Rutkowski, M. (2008) Pricing and trading credit default swaps in a hazard process model. Annals of Applied Probability 18, 2495-2529.

[4] Bielecki, T.R., Jeanblanc, M., and Rutkowski, M. (2009) Credit Risk Modelling Lectures Notes, Osaka University.

[5] Brémaud, P. (1981) Point Processes and Queues: Martingale Dynamics, Springer Verlag.

[6] Cousin, A. and Laurent, J.-P. (2010) Dynamic hedging of synthetic CDO tranches: bridging the gap between theory and practice, to appear as a book chapter, Bloomberg Press.

[7] Cousin, A., Jeanblanc, M. and Laurent, J.-P. (2009) Hedging CDO tranches in a Markovian environment, book chapter, working paper.

[8] Laurent, J.P., Cousin, A. and Fermanian, J.D (2007) Hedging default risk for CDOs in Markovian contagion models, Quantitative Finance, Forthcoming.

[9] Dellacherie, C. (1972) Capacités et processus stochastiques, Springer.

[10] Kusuoka, S. (1999), A remark on default risk models, Adv. Math. Econ. 1, 69-82. 